\title{
Assessment of contraceptive utilization and associated factors among sexually active HIV-positive Indian women: A cross-sectional study from an antiretroviral therapy center
}

\author{
Sneha Sagar ${ }^{\mathrm{a}, 1}$, Aamir Bashir ${ }^{\mathrm{a}, 1}$, Biplab Pal ${ }^{\mathrm{b}}$, Mukhtar Ahmad Dar ${ }^{\mathrm{a}}$, Krishna Pandey ${ }^{\mathrm{c}}$, \\ Krishna Murti ${ }^{\text {a,* }}$ \\ ${ }^{a}$ Department of Pharmacy Practice, National Institute of Pharmaceutical Education and Research, Hajipur, 844102, Bihar, India \\ ${ }^{\mathrm{b}}$ Department of Pharmacology, Lovely Professional University, Jalandhar, 144411, Punjab, India \\ ${ }^{\mathrm{c}}$ Division of Clinical Medicine, Rajendra Memorial Research Institute of Medical Sciences (Indian Council of Medical Research), Agamkuan, Patna, 800007, Bihar, India
}

\section{A R T I C L E I N F O}

\section{Keywords:}

HIV

Reproductive women

Contraception

Family planning

HAART

\begin{abstract}
A B S T R A C T
Background: HIV/AIDS epidemic is one of the most serious global public health problems, more particularly seen in low and middle-income countries. Females contribute around $40 \%$ of total HIV cases in India who predominantly belong to fertile age group. This study aimed to assess the contraceptive utilization patterns and factors affecting the HIV-positive Indian women.

Methods: A cross-section of 400 confirmed HIV-positive women aged between 18 and 45 years were recruited. All the relevant information including socio-demographic and anthropometric details were collected from the participants. All other needed parameters were recorded from patients file. The SPSS ver. 21 was used to perform statistical analysis.

Results: The mean age of the participants was found to be $29.53 \pm 5.35$ years. The majority of women were housewives (97\%), Illiterate (58.8\%) and $90.5 \%$ had a monthly family income under 10,000 INR. Maximum $(82.8 \%)$ of HIV transmission was found through unprotected sexual routes while $68.8 \%$ of patients were on HAART. Male condoms were highly utilized whereas $44.3 \%$ of participants never used any methods. Percentage of consistent contraceptive use was highest when patients were educated to graduate level $(66.7 \%)$, aware of contraception methods (49.8\%) and who have a monthly income more than 20,000 INR (66.7\%).

Conclusion: This study gives a real picture of the contraceptive utilization among HIV-infected women from rural regions of India. The study anticipates the immediate needs in healthcare sector to improve awareness of patients regarding modern methods of contraception and effective family planning strategies.
\end{abstract}

\section{Introduction}

Human Immunodeficiency virus/Acquired immune deficiency syndrome (HIV/AIDS) epidemic is the most serious global public health problem, more particularly seen in low and middle-income countries. ${ }^{1,2}$ The different sections of the population who remain at high risk include, Female sex workers (FSW), Men having sex with men (MSM), Transgenders, Injecting drug users (IUD), Truckers and Migrants. ${ }^{3}$ Globally, there are more than 37 million people living with HIV where 1.8 million new cases were added in 2018. ${ }^{4}$ National Aids Control Organization of India (NACO) has reported 2.1 million patients with a prevalence of
$0.26 \%$ for HIV where females contribute around $40 \%$ of total HIV infections predominantly from fertile age group. ${ }^{5}$ The overall national prevalence is seen steadily declining from last two decades as $0.38 \%$ in $2001-03,0.34 \%$ in $2007,0.28 \%$ in 2012 and $0.26 \%$ in 2017 in both genders. This decline has resulted due to some major advances in health care services provided to HIV-patients, most importantly the rapid expansion of access to antiretroviral therapy (ART) centers since 2002 in the country, where patients can easily go for HIV testing, counselling and treatment. ${ }^{6}$ In Eastern Indian state Bihar, the prevalence is estimated between 0.21 and $0.25 \%$ and the infection continues as a major public health challenge, where only $44 \%$ of active cases are seen

\footnotetext{
* Corresponding author. Department of Pharmacy Practice, National Institute of Pharmaceutical Education and Research, Hajipur, 804102, India.

E-mail address: krishnamurti74@gmail.com (K. Murti).

1 S. Sagar and A. Bashir contributed equally to this work as first authors.
} 
registered at different ART centers. ${ }^{5}$

HIV/AIDS is still considered as one of the most life-threatening diseases especially with fertile women. Importantly, as compared to young men, the young reproductive women have a 4-7-fold increased risk of getting HIV infection. ${ }^{7}$ This viral infection impairs and destroys the normal functioning of the immune system and individual gradually becomes immune-deficient. ${ }^{8}$ After the introduction of highly active antiretroviral therapy (HAART), there has been a reduction in both morbidity and mortality among HIV positive patients and also a significant change in thought is seen to consider HIV/AIDS as a long-term but manageable condition. ${ }^{9}$ Genuinely, like other fertile age women, sexually active HIV positive women also wish a childbearing and parenthood in their life. They also desire to have safe and effective contraception to prevent unwanted pregnancies, prevention from sexually transmitted infections (STIs), and infection of HIV to their sexual partners. However, to utilize a contraceptive method by Indian women, several factors influence the acceptance or denial such as, personal and partner's choice, available methods and services, cultural and religious beliefs and possible adverse effects of contraception. ${ }^{10}$ Therefore, an ideal contraceptive approach needs to be safe, effective, economical, user-friendly and should provide protection from both HIV-transmission and unintended pregnancy.

Currently, several methods of contraception are used by Indian women, such as hormonal [pills, vaginal ring, injections, intrauterine contraceptive device (IUDs)] and non-hormonal methods (copper IUDs, condoms, vaginal sponges). ${ }^{11}$ Among available methods, oral contraceptives, IUD and sterilization are seen highly effective but none of the methods show ability to prevent HIV transmission. ${ }^{12}$ So, a combined approach with appropriate ART therapy and a desired contraceptive method can help in impeding the high prevalence of HIV transmission. In this backdrop, we aimed to assess the prevalence and pattern of contraception methods among HIV positive women who were sexually active and receiving highly active antiretroviral therapy at ART Center in Patna, Bihar.

\section{Material and methods}

\subsection{Study design}

This was a hospital-based cross-sectional study conducted at the antiretroviral therapy center of Rajendra Memorial Research Institute of Medical Sciences-Indian Council of Medical Research (RMRIMS-ICMR) Patna, Bihar. The study protocol was approved by the Institutional Ethics Committee of Rajendra Memorial Research Institute of Medical Sciences. The study was carried out as per the declaration of Helsinki and its amendments. ${ }^{13}$ From all study participants, written informed consent were obtained before administering the questionnaire. The aim and objectives of the study were explained to the participants and were also assured for their confidentiality and anonymity of data. STROBE guidelines were followed while writing manuscript for strengthening the reporting of observational studies in epidemiology. ${ }^{14}$

\subsection{Sample size}

A simple random sampling technique was used to enrol the study subjects. The sample size was calculated by using Open Epi software Version 3.01. ${ }^{15}$ The overall prevalence of contraceptive utilization was taken as $54.7 \%$ among HIV positive patients in India, as reported by a previous study. ${ }^{16}$ On the precision of $5 \%$ at $95 \%$ confidence level, the required sample size was found to be 382 . To further determine the feasibility of this study, a pilot study was performed with 50 enrolled patients and after considering a $5 \%$ non-response rate, the final sample size was taken to 400 .

\subsection{Study participants}

A total of 442 confirmed HIV/AIDS female patients aged between 18 and 45 years who were regularly visiting the ART center of RMRIMS were enrolled in this study. However, only 400 enrolled patients completed the study procedures giving a response rate of $90.49 \%$. The diagnosis was established after the pathological examination of subjects following WHO recommendations (detection of anti-HIV antibodies in serum/plasma.). ${ }^{17}$ The participants with any other concurrent condition like, known infertility, any other STDs, or living with mental illness were excluded from the study. We excluded pregnant women or widows from the study and also patients who have not given their consent and not completed data or who left from the interview.

\subsection{Data collection}

A pretested semi-structured questionnaire was used to collect relevant information from the participants. The questionnaire was prepared in English and then translated into the local language (Hindi). The interview was arranged individually to all enrolled patients for collecting the data. The questionnaire included socio-demographic characteristics (age, marital status, occupation, income, education) and clinical features (CD4 counts, information on ART), sexual activity status, contraceptives choice, and use. A trained interviewer was assigned to administer the questionnaire to ensure completeness and consistency of collected data.

\subsection{Data analysis}

All the data were processed in Microsoft Excel spreadsheets and further analysis was done by using the SPSS software (Version 21; CDC Atlanta, USA). Socio-demographic variables were analyzed by using descriptive statistics. Frequencies and percentages were compared for categorical variables using Pearson's chi-square $\left(\chi^{2}\right)$ test. The continuous variables were shown as mean \pm Standard Deviation (SD). All tests were two-tailed and differences were considered as statistically significant if the $\mathrm{p}$-value was $<0.05$. $^{18}$

\section{Results}

A total of four hundred participants were recruited in this study after meeting all the eligibility requirements. The mean $( \pm \mathrm{SD})$ age of the participants was $29.53 \pm 5.35$ years, ranging from 18 to 45 years. Most of the respondents (45\%) were in the age of 25-31 years and age group of $39-45$ had the least portion (4.3\%). With regard to marital status among infected women, $96 \%$ were married and $4 \%$ were unmarried. Regarding the occupation and education status of participants, most of them were housewives (97\%) and around 59\% were illiterates. Majority of enrolled patients (90.5\%) had a monthly family income under 10,000 INR (Indian rupee). Details of demographic data of the participants are shown in Table 1.

The status of diagnosis, transmission, and treatment of participants is depicted in Table 2 . About $50 \%$ of the patients were diagnosed in the last 3 years, amongst them maximum $(68.8 \%)$ were on HAART therapy. More than $80 \%$ of the enrolled patients had admitted to have unprotected sex whereas, transmission from mother to child and after using IUD method of contraception was found to be negligible. Out of the 400 participants interviewed, $301(75.2 \%)$ had CD4 count more than 350 cell $/ \mathrm{mm}^{3}$.

Male condoms were highly utilized for contraception amongst all the methods while $44.3 \%$ of participants did not use any contraceptive method. The prevalence of choosing a dual method or injectable contraceptive was found to be the same as $0.3 \%$. With respect to consistency of contraceptive use, $53.3 \%$ of respondents had never used contraception methods consistently; however $31.5 \%$ were using contraception regularly in their social life. The details of contraceptive preference and 
Table 1

Socio-demographic characteristics of enrolled HIV positive women.

\begin{tabular}{lll}
\hline Variable & & $\mathrm{N}=400(\%)$ \\
\hline Age (years) & $18-24$ & $72(18)$ \\
& $25-31$ & $180(45)$ \\
& $32-38$ & $131(32.8)$ \\
Marital status & $39-45$ & $17(4.3)$ \\
& Married & $384(96.0)$ \\
Occupation & Unmarried & $16(4.0)$ \\
& Housewife & $388(97.0)$ \\
& Labor & $6(1.5)$ \\
Education & Private Job & $4(1.0)$ \\
& Sex worker & $2(0.5)$ \\
& Illiterate & $235(58.8)$ \\
& Primary & $99(24.8)$ \\
Monthly income (INR) & Secondary & $48(12)$ \\
& Graduates & $18(4.5)$ \\
& $\leq 10,000$ & $362(90.5)$ \\
& $10,000-20000$ & $35(8.75)$ \\
& $\geq 20,000$ & $3(0.075)$ \\
\hline
\end{tabular}

Abbreviations: INR $=$ Indian rupee

Table 2

Status of diagnosis, transmission and treatment of HIV-infection among enrolled patients.

\begin{tabular}{llll}
\hline Variable & & $\mathrm{N}=400(\%)$ & p- value \\
\hline Years since diagnosed & 0-3 years & $199(49.8)$ & $<\mathbf{0 . 0 0 1}$ \\
& 4-6 years & $142(35.5)$ & \\
& 7-9 years & $54(13.5)$ & \\
Years since HAART started & 10-13 years & $5(1.3)$ & \\
& 0-3 years & $275(68.8)$ & $<\mathbf{0 . 0 0 1}$ \\
& 4-6 years & $107(26.8)$ & \\
Mode of HIV transmission & 7-9 years & $18(4.5)$ & \\
& Blood transfusion & $13(3.3)$ & $<\mathbf{0 . 0 0 1}$ \\
& Heterosexual & $25(6.3)$ & \\
& Mother to child & $2(0.5)$ & \\
& Unprotected sex & $331(82.8)$ & \\
& IUD & $2(0.5)$ & \\
& Unknown & $26(6.5)$ & \\
CD4 count & $<350$ & $91(24.8)$ & $<\mathbf{0 0 0 1}$ \\
& $>350$ & $301(75.2)$ & \\
\hline
\end{tabular}

Abbreviations: HAART $=$ highly active antiretroviral therapy, HIV $=$ human immunodeficiency viruses, CD4 = cluster of differentiation.

persuasion are presented in Table 3 (Fig. 1).

The respondents predominantly (71.8\%) did not wish to have children after diagnosed with HIV however, a low but significant proportion $(28.3 \%)$ of infected women still desired to have a child. To check the infection status of sexual partners amongst the participants, $86.8 \%$ were found HIV positive. A greater proportion of participants (37.5\%) were not aware of contraceptive methods, meanwhile the most of respondents (88.5\%) were sexually active (Table 4$)$.

The association between socio-demographic factors and the use of contraceptives is shown in Table 5. Among the illiterate participants, $58.3 \%$ had never used any method of contraception however, most of

Table 3

Methods and consistency of different contraceptives among enrolled HIV positive women.

\begin{tabular}{llll}
\hline Variable & & $\mathrm{N}=400(\%)$ & $\mathrm{p}$ - value \\
\hline Contraceptive choice & None & $177(44.3)$ & $<\mathbf{0 . 0 0 1}$ \\
& Male condom & $182(45.5)$ & \\
& Dual method & $1(0.3)$ & \\
OCP & $3(0.8)$ & \\
& Abstinence & $36(9.0)$ & \\
& Injectable & $1(0.3)$ & \\
Contraceptive use & Consistent & $126(31.5)$ & \\
& Non-consistent & $61(15.3)$ & \\
& Never & $213(53.3)$ & \\
\hline
\end{tabular}

Current contraceptive method utilization among HIV-positive women

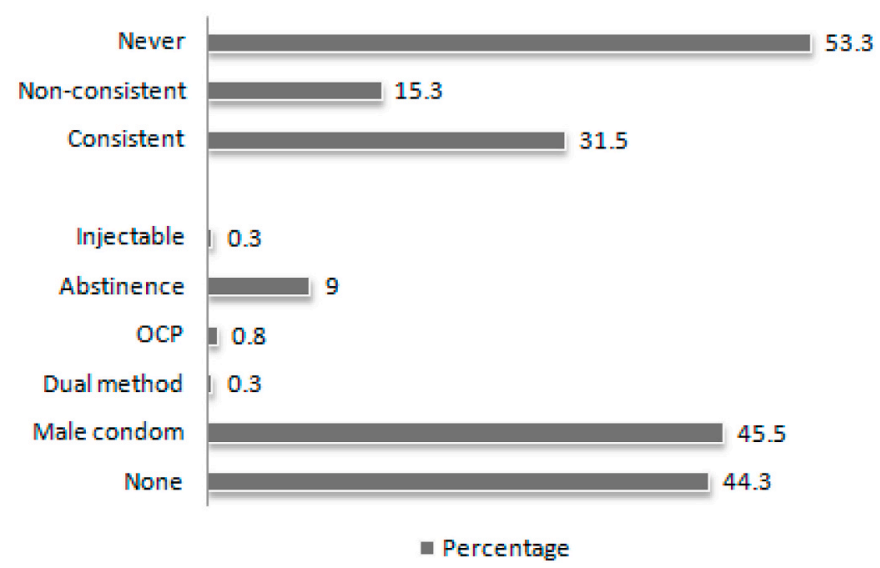

Fig. 1. Current patterns of contraceptive utilization among HIV-positive women attending ART Center Patna.

Abbreviations: $\mathrm{OCP}=$ Oral contraceptive pills.

Table 4

Awareness of contraceptive methods, fertility status and partners infection status among enrolled HIV positive women.

\begin{tabular}{llll}
\hline Variable & & $\mathrm{N}=400(\%)$ & p- value \\
\hline Awareness & Yes & $250(62.5)$ & $<\mathbf{0 . 0 0 1}$ \\
Desire for children & No & $150(37.5)$ & \\
& Yes & $113(28.3)$ & $<\mathbf{0 . 0 0 1}$ \\
Partner status & No & $287(71.8)$ & \\
& Positive & $347(86.8)$ & $<\mathbf{0 . 0 0 1}$ \\
& Negative & $52(13)$ & \\
Sexually active & Unknown & $1(0.3)$ & $<\mathbf{0 . 0 0 1}$ \\
& Yes & $354(88.5)$ & \\
\hline
\end{tabular}

Table 5

The association between socio-demographic variables and use of contraceptives.

\begin{tabular}{|c|c|c|c|c|}
\hline \multirow[t]{2}{*}{ Variable } & \multicolumn{3}{|c|}{ Use of contraceptive n (\%) } & \multirow[t]{2}{*}{$\mathrm{p}$-value } \\
\hline & Consistent & Inconsistent & Never & \\
\hline Education status & & & & 0.007 \\
\hline Illiterate & $63(26.8)$ & 35 (14.9) & $137(58.3)$ & \\
\hline Primary & $31(31.3)$ & $18(18.2)$ & $50(50.5)$ & \\
\hline Secondary & $20(41.7)$ & $5(10.4)$ & $23(47.9)$ & \\
\hline Graduates & $12(66.7)$ & $3(16.7)$ & $3(16.7)$ & \\
\hline Marital status & & & & 0.199 \\
\hline Married & $123(32.0)$ & $60(15.6)$ & $201(52.3)$ & \\
\hline Unmarried & $3(18.8)$ & $1(6.2)$ & $12(75.0)$ & \\
\hline Occupation & & & & 0.154 \\
\hline Housewife & $119(30.7)$ & $60(15.5)$ & 209 (53.9) & \\
\hline Labor & $2(33.3)$ & $1(16.7)$ & $3(50.0)$ & \\
\hline Private Job & $4(100.0)$ & $0(0)$ & $0(0)$ & \\
\hline Sex worker & $1(50.0)$ & $0(0)$ & $1(50.0)$ & \\
\hline Awareness & & & & $<0.01$ \\
\hline Yes & $124(49.8)$ & $56(22.5)$ & $69(27.7)$ & \\
\hline No & $2(1.3)$ & $5(3.3)$ & $144(95.4)$ & \\
\hline Partner status & & & & $<0.01$ \\
\hline Positive & $95(27.4)$ & $58(16.7)$ & $194(55.9)$ & \\
\hline Negative & $30(57.7)$ & $3(5.8)$ & $19(36.5)$ & \\
\hline Unknown & $1(100.0)$ & $0(0)$ & $0(0)$ & \\
\hline Monthly income & & & & 0.666 \\
\hline$\leq 10,000$ & $112(30.9)$ & 57 (15.7) & $193(53.3)$ & \\
\hline $10,000-20000$ & $12(34.3)$ & $4(11.4)$ & $19(54.3)$ & \\
\hline$\geq 20,000$ & $2(66.7)$ & $0(0)$ & $1(33.3)$ & \\
\hline
\end{tabular}

Consistent contraceptive use: Regular use of any methods whenever having intercourse, Inconsistent contraceptive use: Not being regular, using sometimes and missing sometimes The p-value $<0.05$ represents the significant differences between the different subgroups for the variable. 
the patients $(66.7 \%)$ with higher education showed a consistent contraceptive use. In both married and unmarried groups, a higher proportion (52.3\% and $75.0 \%$ respectively) of patients was not using any method of contraception. The majority of participants were housewives and most among them had also never used contraception. $49.8 \%$ of participants were consistently using contraceptives among the patients aware of contraceptives however, a majority (95.4\%) who had no awareness regarding contraception had never used any contraception method. In respondents having HIV positive partners, a significant number $(55.9 \%)$ had never used contraceptives however, $57.7 \%$ of patients were on consistent use of contraceptives in the HIV negative partner group. In both $\leq 10,000$ and $10,000-20000$ income groups, more than $50 \%$ of patients had never used contraception.

\section{Discussion}

This study aimed to assess the patterns of contraceptive utilization among sexually active HIV-positive women coming from the general population. These women were visiting the ART center, Patna from different rural areas of Bihar to get the standard antiretroviral therapy. The current study findings highlight the importance of research in this specific infection to develop potential strategies which can overcome the present situation in this region. The overall prevalence of consistent contraceptive utilization in our study patients was found to be $31.5 \%$ which is almost the same (32.65\%) as reported from the general married women population in that region. ${ }^{19}$ While evaluating the association between different socio-demographic variables and the use of contraceptives, we found a greater variability with the previous literature. Present study findings show that $62.5 \%$ of infected women were aware of the contraceptive methods, however, only $46.7 \%$ were using any of the contraceptive methods to prevent pregnancy. In comparison to these findings, one previous study from a metropolitan city Mumbai has shown higher level of awareness (above 90\%) and use of contraception (over 70\%) among HIV positive women. ${ }^{20}$ Additionally authors have documented that majority of the women were educated (89\%) and also employed (52\%).

In our study participants with no education, $58.3 \%$ had never used contraceptives however, a consistent contraceptive use was observed in most of the patients $(66.7 \%)$ with higher education. In contrast to a former report from India, HIV infected women from Kerala have shown a higher contraceptive utilization (58.1\%) as compared to our study participants. ${ }^{21}$ The authors also put forward that women in Kerala have a better educational and reproductive health status with more than 18 years of median age at marriage. The present study findings primarily highlight the importance of education and awareness among this particular section of the population to prevent HIV infection and its possible transmission.

Education, awareness about contraception, occupation, family income, desire to have children and partner's HIV status were the main factors associated with pattern of contraceptive choice and utilization. Among the study participants, the preferred choice of contraception was male condom (45.5\%) while OCP (oral contraceptive pills), injectable contraceptives and IUCD (Intra-Uterine Contraceptive Device) utilization was almost nil $(<1 \%)$. These observations extend the previous data from the same state which has reported a higher number of patients (42.5\%) with no knowledge about any contraceptive devices. ${ }^{22}$ The findings were also matching with the study of Thulaseedharan JV from Kerala who also reported a non-significant use of these reversible contraceptive options in his study group. However, these observations were in opposition to the results from general reproductive-age women in the same region were $8.87 \%$ were using a condom, $51.91 \%$ were using OCP and $38.56 \%$ were using IUCD reported by Vijay Shree et al. ${ }^{19}$ Additionally, one more study from Ethiopia reported that patients were utilizing IUCD $(28.4 \%)$ or male condoms $(26.7 \%){ }^{23}$

More than $90 \%$ of our recruited patients were from a low-income class and most of them had never used contraception. These results predict the income of patients as an important factor to impact the choice and use of any contraception method. In our study, $86.8 \%$ of sexual partners of patients were also HIV positive and a higher proportion $(71.8 \%)$ among them did not wish to have children. These observations were in line with previous reports from Uganda and another large study from various developing countries. ${ }^{24,25}$ However, the findings were conflicting to a previous study suggesting that women living in a couple were more likely to express their desire to have children. The authors suggest the reasons as an increased social pressure over the couple, to get less social importance when living without children in a society. ${ }^{26}$

Complementary to a previous study from north India, ${ }^{27}$ reported $76.8 \%$ of the patients as married, we observed a much higher percentage (96\%) of infected women as married for whom social and sexual life was a major concern. Among the participants, we also found that $97 \%$ were housewives, mostly in the age group of 25-31 years. These estimates predict that the infection is not actually restricted to a specific high-risk population group, however people from the general population predominantly from productive age could also be the infection targets. ${ }^{28}$ Globally, the heterosexual route of HIV transmission is considered as the most common mode of HIV infection. ${ }^{29,30}$ The fact is also supported by many Indian reports, ${ }^{31,32}$ however in our research we found contrarily a maximum percentage $(82.8 \%)$ of women getting the infection due to unprotected sexual intercourse with their partner. These results significantly define the unmet need of awareness among people regarding methods of protection while having sexual activity.

In the current study, more than $80 \%$ of patients were using male condoms among all the contraceptive methods utilized. There are several reasons which favour this contraceptive choice counted as, free availability of condoms in all public healthcare centers, high effectiveness in preventing unwanted pregnancies and its capacity of preventing secondary transmission of infection during sex with a partner of unknown HIV status. The other important perspective might be the lack of awareness about other contraceptive methods which are currently being approved to prevent unwanted pregnancies. Additionally male condoms are preferred and recommended by the healthcare providers due to the fear of possible drug interaction between hormonal contraceptives and antiretroviral drug therapy. ${ }^{33}$

About half of the study patients were diagnosed in the last three years and around $70 \%$ among them had started the HAART therapy. These findings are encouraging in a way that due to the availability of free services and easy access to ART centers in India, poor and needy people are utilizing these healthcare facilities. Such facilities help in preventing them and other vulnerable populations from these deadly infectious diseases. ${ }^{34}$ In addition, patients receiving HAART regularly visit ART centers and remain in touch with professionals who educate and counsel them about strategies to deal with infection. ${ }^{35}$

Among our participants, we found primacy of consistent contraceptive use were highest when they were, highly educated and aware of contraceptives, from higher-income group, diagnosed 7-9 years before however, chances were low when patients partner was also HIV positive. These results were also supported by the findings of multiple studies from India, Kenya, Centrale and Kara regions of Togo, Ethiopia and Malawi. ${ }^{20,21,36-39}$ To summarize, our findings suggest that appropriate programs and policies are needed to empower women with proper information and education about the modern contraceptive methods to prevent HIV infection and its transmission.

\section{Strengths and limitations}

The current study has a number of strengths and limitations which are worth to mention. Strengths included; first, we have followed a random sample selection procedure to enrol the patients so, the study can represent a real-world epidemiological situation. Second, this study was conducted in an economically underdeveloped region of India where a well established reproductive healthcare system needs to be 
developed to prevent its public from such dreadful infections. Third, the results of the study can aid and strongly influence the decision-making process of healthcare professionals in developing standard recommendations to control the ever-increasing HIV infection and its transmission. Our study also consists of several limitations; first, due to small sample size and single-center data collection, it may not represent the whole nation, as there are huge cultural disparities across the country which influences the contraceptive utilization. Second, in the study, only HIV positive females were enrolled so we couldn't determine the awareness level of the male population about contraception methods who are indifferent infection targets. Third, authors were unable to address the multicollinearity among the predictors of contraceptive utilization as all of them were categorical. Finally, our study design was cross-sectional and the results do not allow causal interpretations.

Therefore a number of regular longitudinal cohort studies are urgently needed to explore the contraceptive utilization and the need for additional reproductive healthcare services. These important steps can help in providing support, education, and awareness to all the high-risk reproductive age women in preventing unwanted pregnancies and infection spread. The diverse available contraceptive choices can further reduce the number of HIV-positive births and infection transmission to sexual partners.

\section{Conclusion}

This study gives a better comprehension of patterns of the contraceptive utilization and reproductive health status of HIV-positive women living in rural regions of India. The level of education, knowledge of diverse contraceptive methods, occupation, income status, desire for children and partner's HIV status were the main influencing factors determining contraceptive preferences among included patient population. Only male condoms were highly utilized so people need to be educated about the importance of the different modern methods of contraception also. Efforts need to intensify at public health facilities by the policy-makers to encourage contraceptive utilization for improving family planning strategies.

\section{Ethics statement}

The study protocol was approved by the Institutional Ethics Committee of Rajendra Memorial Research Institute of Medical Sciences (RMRIMS), Patna (Reference No. 23/RMRI/EC/2018).

\section{Funding}

This study was conducted without any specific grant from any funding agency, commercial or not-for-profit sectors.

\section{Authors contribution}

Conceived and designed the experiments: SS, AB, BP, MAD, KP, KM. Collection of data: SS, KP.

Analyzed the data: SS, AB, MAD, BP.

Wrote the paper: SS, AB, BP, MAD, KP, KM.

\section{Declaration of competing interest}

There is no known conflict of interest associated with this publication.

\section{Acknowledgements}

Authors would like to thank all the ART Center staff of RMRIMSPatna who helped in all the stages of patient recruitment.

\section{References}

1 Idele P, Hayashi C, Porth T, Mamahit A, Mahy M. Prevention of mother-to-child transmission of HIV and paediatric HIV care and treatment monitoring: from measuring process to impact and elimination of mother-to-child transmission of HIV. AIDS Behav. 2017 Jul 1;21(1):23-33.

2 World Health Organization. Number of People (All Ages) Living with HIV Estimates by WHO Region.

3 Gupta AK, Saran R. Detection of antibodies to HIV-infection among high risk groups in Bihar (India). Indian J Publ Health. 1993;37(2):54-56.

4 Patrikar S, Kachroo K, Sharma J, et al. A systematic review and cost-effectiveness analyses of the new World Health Organization guidelines for the treatment of HIVpositive adults in India. Med J Armed Forces India. 2019 Jan 1;75(1):31-40.

5 National aids control organization of India (NACO) annual report 2016-17. Available from: http://naco.gov.in/sites/default/files/NACO\%20ANNUAL\%20REPORT\%2020 16-17.pdf.

6 Kulkarni MB, Bhalerao MM. Effect of ante retroviral therapy on cd4 count in people living with HIV/AIDS. Indian J Appl Res. 2019 Aug 1;9(4).

7 Simon V, Ho DD, Karim QA. HIV/AIDS epidemiology, pathogenesis, prevention, and treatment. Lancet. 2006 Aug 5;368:489-504 (9534).

8 Duggal S, Chugh TD, Duggal AK. HIV and malnutrition: effects on immune system. Clin Dev Immunol. 2012;2012:784740. https://doi.org/10.1155/2012/784740. Epub 2012 Jan 2. PMID: 22242039; PMCID: PMC3254022.

9 Montaner JS, Lima VD, Harrigan PR, et al. Expansion of HAART coverage is associated with sustained decreases in HIV/AIDS morbidity, mortality and HIV transmission: the "HIV Treatment as Prevention" experience in a Canadian setting. PloS One. 2014 Feb 12;9(2), e87872.

10 Pandey SM. Correlates of modern contraception practices among married couples in rural area of Hisar (Haryana). Indian J Prev Soc Med. 2011;42(3):274-277.

11 Mozumdar A, Gautam V, Gautam A, et al. Choice of contraceptive methods in public and private facilities in rural India. BMC Health Serv Res. 2019 Dec;19(1):421.

12 Bajwa SK, Bajwa SJ, Ghai GK, Singh K, Singh N. Knowledge, attitudes, beliefs, and perception of the north Indian population toward adoption of contraceptive practices. Asia Pac J Publ Health. 2012 Nov;24(6):1002-1012.

13 General Assembly of the World Medical Association. World Medical Association Declaration of Helsinki: ethical principles for medical research involving human subjects. J Am Coll Dent. 2014;81(3):14.

14 Von Elm E, Altman DG, Egger M, et al. The Strengthening the Reporting of Observational Studies in Epidemiology (STROBE) statement: guidelines for reporting observational studies. PLoS Med. 2007;4(10):e296.

15 Sullivan KM, Dean MA, Soe MM, Mctm M. An Introduction to OpenEpi.

16 New JR, Cahill N, Stover J, Gupta YP, Alkema L. Levels and trends in contraceptive prevalence, unmet need, and demand for family planning for 29 states and union territories in India: a modelling study using the Family Planning Estimation Tool. The Lancet Global Health. 2017 Mar 1;5(3):e350-e358.

17 World Health Organization. Consolidated Guidelines on the Use of Antiretroviral Drugs for Treating and Preventing HIV Infection: Recommendations for a Public Health Approach. World Health Organization; 2016.

18 Sterne JA, Smith GD. Sifting the evidence-what's wrong with significance tests? Phys Ther. 2001;81(8):1464-1469.

19 Shree V, Prasad RR, Kumar S, Sinha S, Choudhary SK. Factors for non-acceptance of contraceptive methods amongst married women of reproductive age group in rural Patna. International Journal Of Community Medicine And Public Health. 2017 May 22;4 (6):1882-1887.

20 Joshi B, Velhal G, Chauhan S, et al. Contraceptive use and unintended pregnancies among HIV-infected women in Mumbai. Indian J Community Med: official publication of Indian Association of Preventive \& Social Medicine. 2015 Jul;40(3):168.

21 Thulaseedharan JV. Contraceptive use and preferences of young married women in Kerala, India. Open Access J Contracept. 2018;9:1.

22 Kumari R, Shahnawaz K. Knowledge, attitude \& practices of medical abortionin women-a longitudinal study from Patna, Bihar. J. Evid. BasedMed. Healthc. 2018;5 (12):1037-1040.

23 Mersha AG, Erku DA, Belachew SA, Ayele AA, Gebresillassie BM, Abegaz TM. Contraceptive use among HIV-positive and negative women: implication to end unintended pregnancy. Contraception and reproductive medicine. 2019 Dec;4(1):3.

24 Pritchett LH. Desired fertility and the impact of population policies. Popul Dev Rev. $1994 ; 20: 1-55$.

25 Nakayiwa S E, Abang B, Packel L, et al. Desire for children and pregnancy risk behavior among HIV-infected men and women in Uganda. AIDS Behav. 2006;10(4 suppl):S95-S104.

26 Jose H, Madi D, Chowta N, et al. Fertility desires and intentions among people living with HIV/AIDS (PLWHA) in Southern India. J Clin Diagn Res: J Clin Diagn Res. 2016 Jun;10(6):OC19.

27 Wig N, Sakhuja A, Agarwal SK, Khakha DC, Mehta S, Vajpayee M. Multi dimensional health status of HIV-infected outpatients at a tertiary care center in north India. Indian J Med Sci. 2008;62:87-97.

28 Yu Bashir, Farhana A, Ahmed J, Saleem SM, Shafi H. Clinico-epidemiological and socio-demographic profile of HIV/AIDS patients diagnosed at a tertiary care centre in kashmir. Microbiology Research Journal International. 2019 Mar 30:1-7.

29 Fauci AS, Lane HC. Human immunodeficiency virus disease. AIDS and related disorders. In: Kasper DL, Fauci AS, Longo DL, Braunwald E, Hauser SL, Jameson JL, eds. Harrison's Principles of Internal Medicine. seventeenth ed. USA: The McGraw-Hill companies, Inc.; 2008:1137-1203.

30 Amballi AA, Ajibola A, Ogun SA, Ogunkolo OF, Salu LO, Oritogun KS. Demographic pattern and haemotological profile in people living with HIV/AIDS in a university teaching hospital. Sci Res Essays. 2007;2:315-318. 
31 Piot P. Joint united nations program on HIV/AIDS (UNAIDS). In: Cancer, AIDS, and Quality of Life. Boston, MA: Springer; 1997:7-10.

32 Shukla Y, Rohit BK, Tiwari R, Kasar PK. Sociodemographic profile of people living with HIV/AIDS attending ART center in a tertiary-care hospital in Central India. Int $J$ Med Sci Publ Health. 2015 Oct 1;4(10):1464-1468.

33 Nanda K, Stuart GS, Robinson J, Gray AL, Tepper NK, Gaffield ME. Drug interactions between hormonal contraceptives and antiretrovirals. AIDS. 2017 Apr 24;31(7):917.

34 Fowler MG, Qin M, Fiscus SA, et al. Benefits and risks of antiretroviral therapy for perinatal HIV prevention. $N$ Engl J Med. 2016 Nov 3;375(18):1726-1737.

35 Zhao Y, McGoogan JM, Wu Z. The benefits of immediate ART. J Int Assoc Phys AIDS Care. 2019 Mar 4;18, 2325958219831714.

36 Okoli ML, Alao S, Ojukwu S, Emechebe NC, Ikhuoria A, Kip KE. Predictive and spatial analysis for estimating the impact of sociodemographic factors on contraceptive use among women living with HIV/AIDS (WLWHA) in Kenya: implications for policies and practice. BMJ open. 2019 Jan 1;9(1), e022221.

37 Yaya I, Patassi AA, Landoh DE, et al. Modern contraceptive use among HIV-infected women attending HIV care centres in Togo: a cross-sectional study. BMJ open. 2018 Apr 1;8(4), e019006.

38 Worke MD, Bezabih LM, Woldetasdik MA. Utilization of contraception among sexually active HIV positive women attending art clinic in University of Gondar Hospital: a hospital based cross-sectional study. BMC Wom Health. 2016 Dec;16(1): 67.

39 Habte D, Namasasu J. Family planning use among women living with HIV: knowing HIV positive status helps-results from a national survey. Reprod Health. 2015 Dec;12 (1):41. 